\title{
Surgically Important Relationships of Recurrent and Nonrecurrent Laryngeal Nerves to the Coexisting Variant Vessels
}

\author{
Nabil Eid, Yuko Ito, Yoshinori Otsuki \\ Department of Anatomy and Cell Biology, Division of Life Sciences, Osaka Medical College, Osaka, \\ Japan \\ Email: nabil@art.osaka-med.ac.jp, nabileidm@yahoo.com
}

Received 5 November 2014; revised 6 December 2014; accepted 17 December 2014

Copyright (C 2015 by authors and Scientific Research Publishing Inc.

This work is licensed under the Creative Commons Attribution International License (CC BY).

http://creativecommons.org/licenses/by/4.0/

(c) (i) Open Access

\begin{abstract}
The retroesophageal right subclavian artery may be associated with multiple neurovascular variations, which may impact various cervicothoracic surgeries. During the dissection of 27 cadavers, the authors detected a retroesophageal right subclavian artery in old man arising from distal aortic arch, crossed ventrally by left recurrent laryngeal nerve and dorsally by thoracic duct. On the right side, the aberrant subclavian artery was associated with nonrecurrent laryngeal nerve crossing a large tortuous inferior thyroid artery at multiple intersection points. Importantly, the nonrecurrent laryngeal nerve gave rise to extra laryngeal branch passing over Zuckerkandl's tubercle of thyroid gland before its termination into the larynx. The relationships of the aberrant subclavian artery in the present case to thoracic duct, recurrent and nonrecurrent laryngeal nerves may have clinical relevance to various operations such as thyroidectomy, esophagectomy and surgical correction of the aberrant vessel. Therefore, these relationships should be well known by surgeons for prevention of iatrogenic damage of essential neurovascular structures during various surgeries.
\end{abstract}

\section{Keywords}

Inferior Thyroid Artery, Nonrecurrent Laryngeal Nerve, Retroesophageal Subclavian Artery, Zuckerkandl's Tubercle

\section{Introduction}

The retroesophageal right subclavian artery (RERSA) is a rare variation $(0.5 \%$ to $1 \%)$ directly related to the

How to cite this paper: Eid, N., Ito, Y. and Otsuki, Y. (2015) Surgically Important Relationships of Recurrent and Nonrecurrent Laryngeal Nerves to the Coexisting Variant Vessels. Forensic Medicine and Anatomy Research, 3, 16-19.

http://dx.doi.org/10.4236/fmar.2015.31003 
esophagus with surgical implications. It is usually associated with right nonrecurrent laryngeal nerve (NRLN) and abnormal cerebral arteries and thoracic duct (TD). The embryological origin of the RERSA is related to abnormal involution of the fourth right aortic arch. Studies regarding the relationship between the right NRLN and the associated variant inferior thyroid artery (ITA) and Zuckerkandl's tubercle (ZT) of thyroid gland in cases of RERSA are very few in spite of the surgical relevance to thyroid surgery [1]-[4]. In addition, the relationships of RERSA to left recurrent laryngeal nerve (LRLN) and TD are not well investigated, although such relationships may impact various surgeries such as correction of the aberrant vessel and various operative techniques for esophageal cancer [5]-[9].

\section{Case Report}

During the dissection of 27 cadavers, the authors observed abnormal branching pattern of aortic arch (AA) in old man (Figure 1, lower inset); the AA branched into right common carotid artery (RCCA), left common carotid artery and left subclavian artery. The vertebral artery arose from left subclavian artery with normal morphology (grasped by forceps) (Figure 1, lower inset). Retraction of AA and RCCA revealed a RERSA crossed ventrally by LRLN at its origin from AA. Moreover, deeper dissection revealed the TD passing deep to the aberrant artery (Figure 1, upper inset). The aberrant artery gave rise to large thyrocervical trunk and from the latter artery a large tortuous ITA originated and was crossed at multiple proximal and distal levels by NRLN (Figure 1). Importantly, the intersection points between the ITA and NRLN was close to the origin of NRLN and its laryngealentry just above ZT. Moreover, the NRLN gave rise to extralaryngeal branch crossing superficial to ZT before its termination. The right vertebral artery was abnormally kinked close to the crossing point by right vagus nerve (Figure 2).

\section{Discussion}

The NRLN is more prone to injury in thyroid surgery, therefore, preoperative detection and intra operative neuromonitoring of this nerve may be a must [3] [4]. The preoperative prediction of NRLN is based on ultrasonographic and tomographic detection of RERSA, in addition to other methods [3] [10] [11]. The intraoperative identification of NRLN depends on its relationship to thyroid vessels: In type 1, the nerve runs with superior thyroid artery, in type $2 \mathrm{~A}$, the nerve runs transversely over the trunk of ITA while in type $2 \mathrm{~B}$, the nerve crosses the ITA at one intersection level [3] [4]. However, in the present case, the tortuous ITA was crossed by NRLN at multiple levels of intersections; a unique pattern different from type $2 \mathrm{~B}$ and could be of surgical importance asthis nerve could be accidentally injured during its exposure and ligation of ITA in thyroidectomy. Furthermore, the NRLN in the present case entered the larynx just above ZT before giving its rare extra laryngeal branch; the latter branch could be damaged during surgical exposure of NRLN in various neck surgeries as lymph node re-

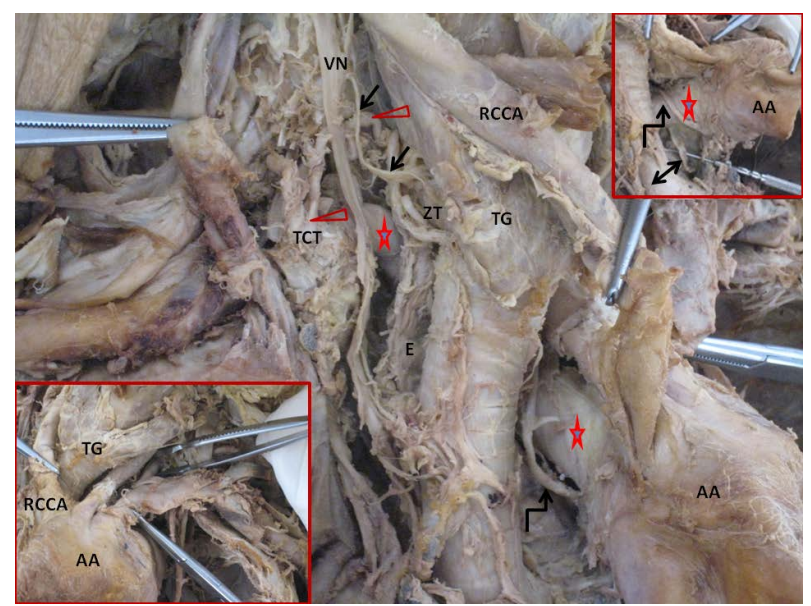

Figure 1. A nonrecurrent laryngeal nerve (arrows) crossing a tortuous inferior thyroid artery (red arrow heads) arising from the right thyrocervical trunk (TCT). The red stars mark the retroesophageal right subclavian artery. The left recurrent laryngeal nerve and thoracic duct are indicated by broken and double head arrows, respectively (upper inset). AA, aortic arch; TG, thyroid gland; RCCA, right common carotid artery; VN, vagus nerve; E, esophagus; ZT, Zuckerkandl's tubercle. 


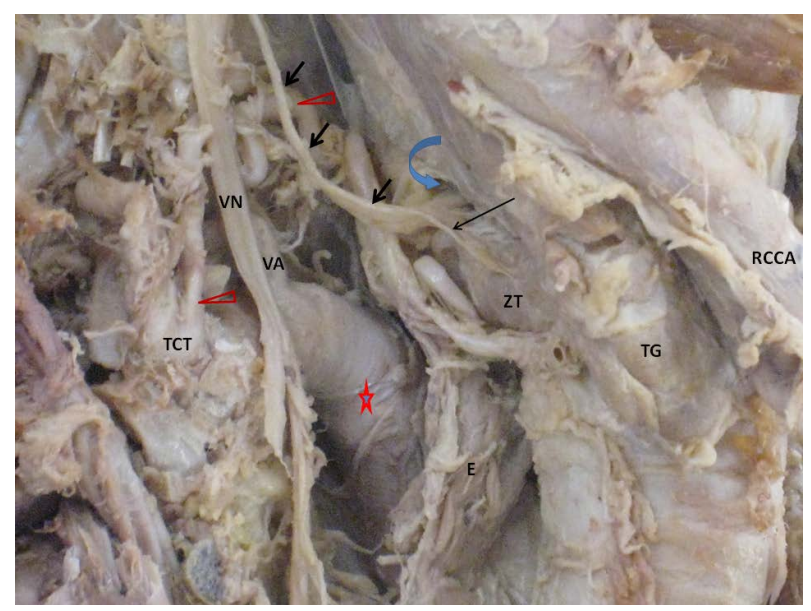

Figure 2. Multiple intersection points of nonrecurrent laryngeal nerve (short arrows) with inferior thyroid artery (red arrow heads) at various proximal and distal levels. The red star marks the retroesophageal right subclavian artery. The curved blue arrow indicates the laryngeal entry of left recurrent laryngeal nerve. Long arrow marks the extralaryngeal branch of nonrecurrent laryngeal nerve. VN, vagus nerve; TG, thyroid gland, ZT, Zuckerkandl's tubercle; VA, vertebral artery; RCCA, right common carotid artery; TCT, thryocervical trunk.

section for esophageal cancer and thyroidectomy [6] [7] [12]. The relationship of TD and LRLN to the RERSA in the present case may be of surgical importance because the LRLN and TD could be injured during anastomosis of the divided RERSA to the ascending aorta or the RCCA on surgical correction of RERSA [5]. In addition, the duct, LRLN and NRLN should be secured during surgical treatment of esophageal cancer associated with RERSA. Moreover, the RERSA could be injured during video-assisted thoracoscopic esophagectomy. Therefore, preoperative diagnosis of RERSA in cancer esophagus patients based on symptoms as dysphagia lusoria or imaging methods may help to avoid injury of the aberrant artery [6]-[9]. The V1 segment of the right vertebral artery in the present case was kinked and therefore, it could impact the diagnosis and treatment of cerebral ischemia [13].

\section{Conclusion}

To the best of our knowledge, the present case is the first showing the coexistence of RERSA with NRLN, variant ITA and kinked vertebral artery on right side, in addition to the relationships of the RERSA to TD and LRLN on left side. Moreover, the surgical anatomy of the present case was briefly discussed.

\section{References}

[1] Kobayashi, M., Yuta, A., Okamoto, K. and Majima, Y. (2007) Non-Recurrent Inferior Laryngeal Nerve with Multiple Arterial Abnormalities. Acta Oto-Laryngologica, 127, 332-336. http://dx.doi.org/10.1080/00016480600801415

[2] Obaid, T., Kulkarni, N., Pezzi, T.A., Turkeltaub, A.E. and Pezzi, C.M. (2013) Coexisting Right Nonrecurrent and Right Recurrent Inferior Laryngeal Nerves: A Rare and Controversial Entity: Report of a Case and Review of the Literature. Surgery Today, 44, 2392-2396. http://dx.doi.org/10.1007/s00595-013-0800-5

[3] Randolph, G.W. (2013) Surgical Anatomy and Monitoring of the Recurrent Laryngeal Nerve. In: Randolph, G.W., Ed., Surgery of the Thyroid and Parathyroid Glands, Elsevier Inc., 33.

[4] Kamani, D., Potenza, A.S., Cernea, C.R., Kamani, Y.V., Randolph, G.W. (2014) The Nonrecurrent Laryngeal Nerve: Anatomic and Electrophysiologic Algorithm for Reliable Identification. Laryngoscope, Published Online. http://dx.doi.org/10.1002/lary.24823

[5] Atay, Y., Engin, C., Posacioglu, H., Ozyurek, R., Ozcan, C., Yagdi, T., et al. (2006) Surgical Approaches to the Aberrant Right Subclavian Artery. Texas Heart Institute Journal, 33, 477-781.

[6] Sato, H., Tsubosa, Y. and Ugumori, T. (2005) Esophagectomy with Three-Field Lymph Node Dissection for Esophageal Carcinoma with a Nonrecurrent Inferior Laryngeal Nerve. The Japanese Journal of Thoracic and Cardiovascular Surgery, 53, 502-504. http://dx.doi.org/10.1007/s11748-005-0096-5

[7] Takata, O., Kiyozaki, H., Yoshida, T. and Konishi, F. (2007) Nonrecurrent Inferior Laryngeal Nerve in Patients with 
Esophageal Cancer: Report of Two Cases. Esophagus, 4, 41-45. http://dx.doi.org/10.1007/s10388-006-0099-Z

[8] Pantvaidya, G.H., Mistry, R.C., Ghanekar, V.R., Upasani, V.V. and Pramesh, C.S. (2005) Injury of an Aberrant Subclavian Artery: A Rare Complication of Video Assisted Thoracoscopic Esophagectomy. Annals of Thoracic and Cardiovascular Surgery, 11, 35-37.

[9] Kasashima, H., Kubo, N., Ohira, M., Sakurai, K., Toyokawa, T., Tanaka, H., Muguruma, K., Shibutani, M., Yamazoe, S., Kimura, K., Nagahara, H., Amano, R., Ohtani, H., Yashiro, M., Maeda, K. and Hirakawa, K. (2014) Successful Resection of Esophageal Carcinoma with Aberrant Right Subclavian Artery Using Video-Assisted Thoracoscopic Surgery: Report of Two Cases. Anticancer Research, 34, 899-904.

[10] Yetisir, F., Salman, A.E., Çiftçi, B., Teber, A. and Kiliç, M. (2012) Efficacy of Ultrasonography in Identification of Non-Recurrent Laryngeal Nerve. International Journal of Surgery, 10, 506-509. http://dx.doi.org/10.1016/j.ijsu.2012.07.006

[11] Gao, E.L., Zou, X., Zhou, Y.H., Xie, D.H., Lan, J. and Guan, H.G. (2014) Increased Prediction of Right Nonrecurrent Laryngeal Nerve in Thyroid Surgery Using Preoperative Computed Tomography with Intraoperative Neuromonitoring Identification. World Journal of Surgical Oncology, 12, 262. http://dx.doi.org/10.1186/1477-7819-12-262

[12] Gurleyik, E., Dogan, S., Gunal, O. and Pehlivan, M. (2012) The Rare Coincidence: Nonrecurrent Laryngeal Nerve Pointed by a Zuckerkandl's Tubercle. Case Reports in Medicine, 2012, Article ID: 143049. http://dx.doi.org/10.1155/2012/143049

[13] Wagnerova, H. and Goldenberg, Z. (2013) Transient Ischemic Attack in the Vertebro-Basilar Circulation Due to a Hemodynamically Significant Variation-Kinking of the Extracranial Section of the Left Vertebral Artery. Bratislavske Lekarske Listy, 114, 590-594. 
Scientific Research Publishing (SCIRP) is one of the largest Open Access journal publishers. It is currently publishing more than 200 open access, online, peer-reviewed journals covering a wide range of academic disciplines. SCIRP serves the worldwide academic communities and contributes to the progress and application of science with its publication.

Other selected journals from SCIRP are listed as below. Submit your manuscript to us via either submit@scirp.org or Online Submission Portal.
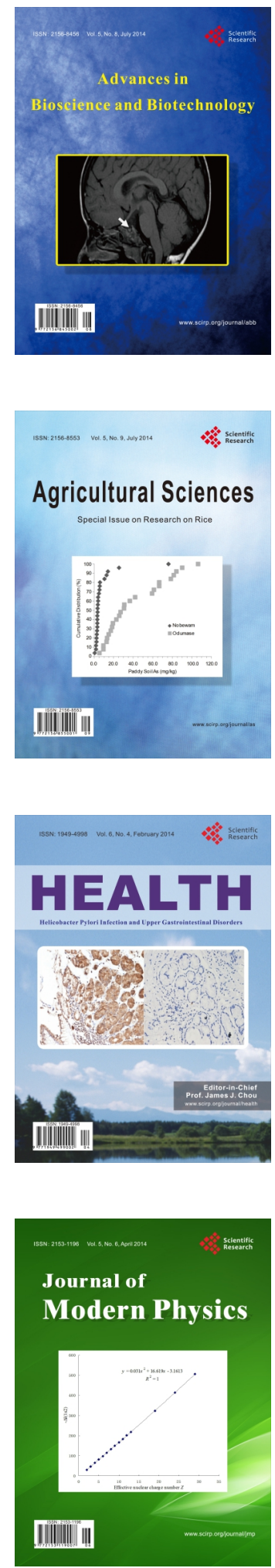
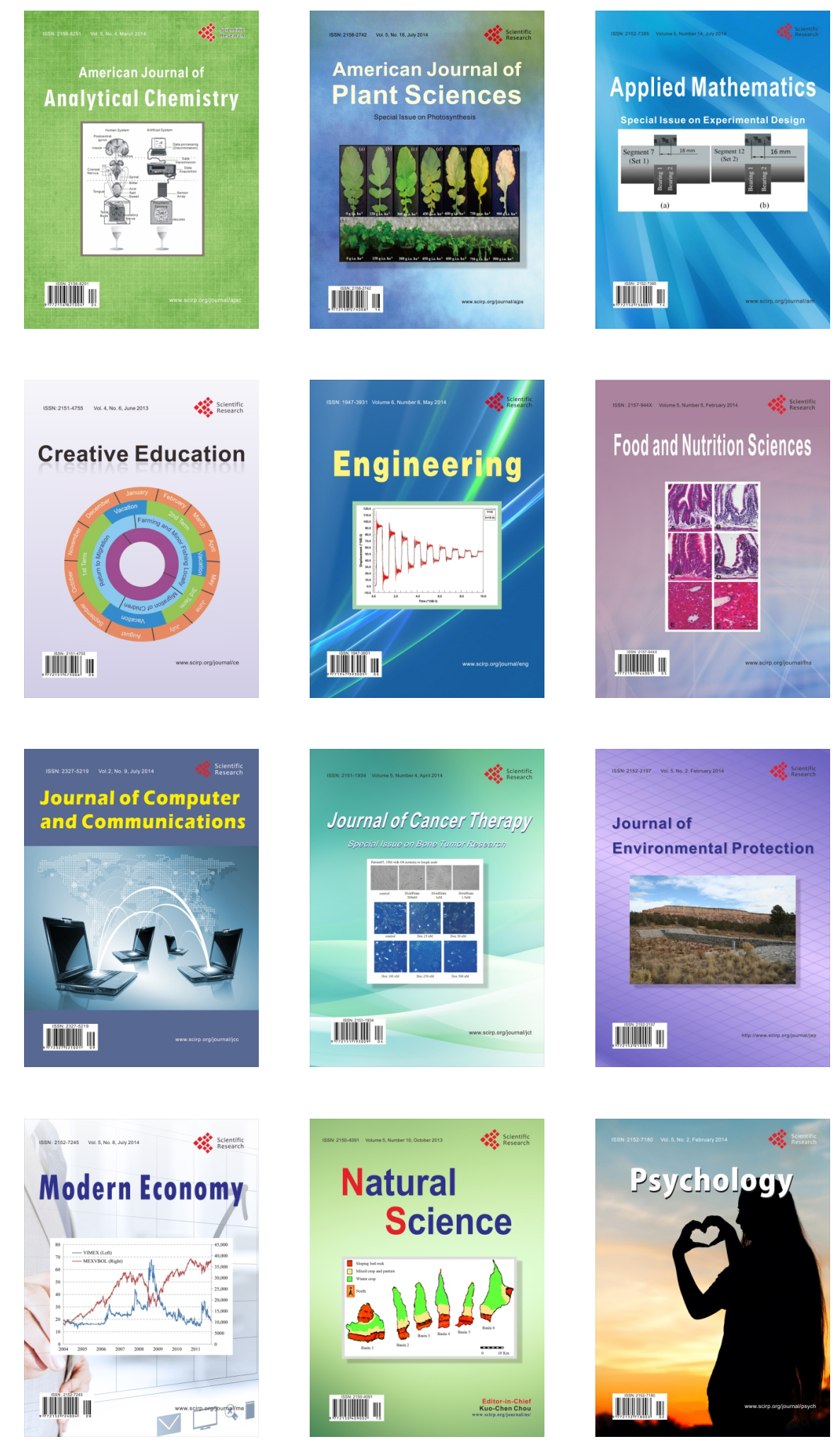